Ecology, 0(0), 2019, e02768

(C) 2019 by the Ecological Society of America

\section{Does polymorphism make the Asiatic golden cat the most adaptable predator in the Eastern Himalayas?}

\author{
Sahil Nijhawan, ${ }^{1,2,4}$ Iho Mitapo, ${ }^{3}$ Jibi Pulu, ${ }^{3}$ \\ Chris Carbone $^{2}$, and Justin Marcus Rowcliffe ${ }^{2}$
}

Manuscript received 18 December 2018; revised 10 April 2019; accepted 25 April 2019. Corresponding Editor: John Pastor.

${ }^{1}$ Department of Anthropology, University College London, London WC1H 0BW United Kingdom.

${ }^{2}$ Institute of Zoology, Zoological Society of London, Regent's Park, London NW1R 4RY United Kingdom.

${ }^{3}$ Dibang Adventures, Lower Dibang Valley, Roing, Arunachal Pradesh 792110 India.

${ }^{4}$ E-mails: s.nijhawan@ucl.ac.uk and sahil.nsit@gmail.com

Citation: Nijhawan, S., I. Mitapo, J. Pulu, C. Carbone, and J. M. Rowcliffe. 2019. Does polymorphism make the Asiatic golden cat the most adaptable predator in the Eastern Himalayas? Ecology 00(00):e02768. 10.1002/ecy. 2768

Key words: Arunachal Pradesh; Asiatic golden cat; camera trap; Catopuma temminckii; coloration phenotypes; habitat segregation; Himalayas; melanism; polymorphism.

Some wild felines have a diverse range of coat colors; others do not. Jaguars and leopards, for instance, come in spotted and melanistic forms, but tigers are always striped and lions always beige. Smaller cats, like clouded leopards, marbled cats, and ocelots are almost always patterned in the same way, whereas jaguarundis, oncillas, and golden cats occur in several different colors and patterns. Does a greater range of coat colors and patterns make some smaller cats more ecologically adaptable than others? We explore this question for the Asiatic golden cat in light of data from an extensive camera trap study in Northeast India that has revealed a previously unknown color form and potentially new insights about advantages of polymorphism in this species.

The Asiatic golden cat Catopuma temminckii (IUCN Red List Near Threatened) is the largest of the group of smaller Asian felines. It is distributed from eastern Nepal through Northeast India, Myanmar, Thailand, and China to Sumatra, Indonesia where it is found from sea level to above 4,000 $\mathrm{m}$ in diverse habitats (McCarthy et al. 2015). Four color forms have been commonly recorded: golden (fox red to ashy red to golden brown), gray, melanistic, and spotted, otherwise called ocelot (McCarthy et al. 2015). An additional color form, cinnamon, has been described by Sunquist and Sunquist (2002), but its occurrence and distribution have not been verified yet. Golden cats prey on a wide range of species from smaller animals such as rodents, snakes, lizards, and birds to medium-sized prey such as muntjac (genus Muntiacus; Sunquist and Sunquist 2002).

From January 2014 to September 2015, we placed single or paired camera units in 223 locations in eight distinct sites in the Dibang Valley district $\left(9,129 \mathrm{~km}^{2}\right)$ of the Indian state of Arunachal Pradesh. The sites were a mix of community forests and Legally protected areas. Because the aim of the study was to estimate densities of all medium-large mammals, we employed two placement strategies: (1) randomized-cameras were placed at nodes of a predetermined $1.5 \times 1.5 \mathrm{~km}$ grid [to meet design requirement of a novel density estimation method, Nijhawan (2018)]; (2) targeted-cameras were placed opportunistically within and around this grid along trails, waterholes, ridgelines, and valley bottoms to maximize capture rates for medium-large carnivores. Each site was surveyed continuously around the clock for 32-68 d. The camera locations spanned an altitudinal range of 535-3,710 $\mathrm{m}$ (see Appendix S1 for further details on methodology).

After over 20 months of fieldwork and 12,671 trap nights, we collected 75 independent photographic records of Asiatic golden cats (an independent detection event is defined as an animal entering and exiting the camera's detection zone; see Appendix S1 for further details). Six different color morphs were photographed - golden, gray, cinnamon, melanistic, ocelot, and a uniquely patterned darker form with tightly spaced rosettes (henceforth, tightly rosetted; Fig. 1). The most commonly detected morph was the default golden form followed by gray and melanistic, whereas the cinnamon morph was photographed just twice in a single site only (potentially indicating rarity). Here, we report the first record of (1) a tightly rosetted form of the species, and (2) an ocelot and cinnamon morph from India. Importantly, Dibang Valley hosts the most diverse range of color morphs of the species ever reported from a single locality.

Earlier studies from India (Sikkim), Nepal, and Bhutan reported gray and melanistic morphs exclusively from higher altitudes (above 2,500 m), with the gray morph, in particular, at much higher altitudes of 3,900 m (Ghimirey and Pal 2009, Bashir et al. 2011, Jigme 2011). In Bhutan, ocelot morphs have only been 


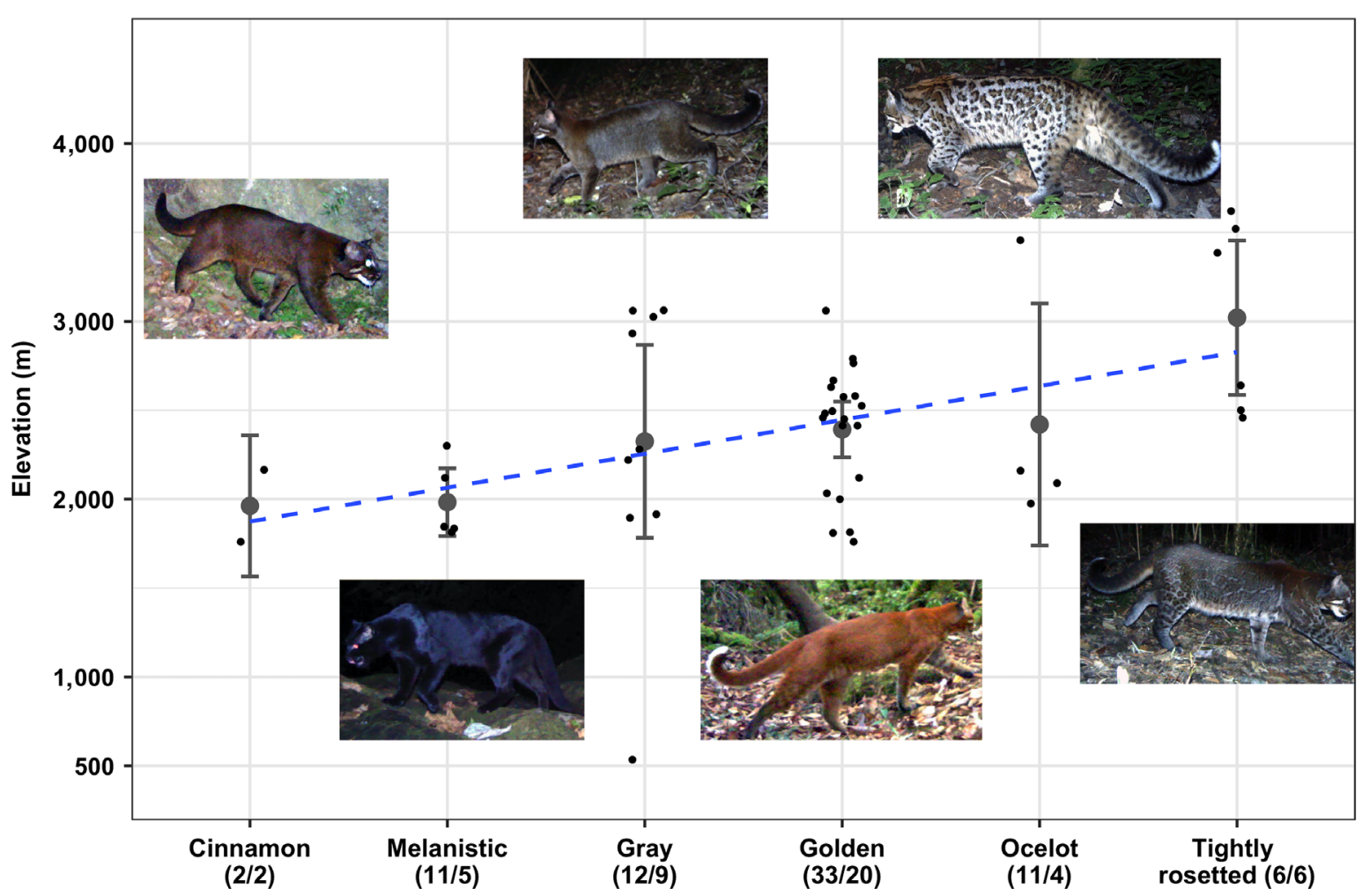

Fig. 1. Altitudinal records of Asiatic golden cat morphs in Dibang Valley. Black dots (jittered for clarity): independent detection records; gray circles and error bars: mean elevations based on single observations at camera traps with $95 \%$ confidence intervals; blue dashed line: least-squares trend line through the means; values morph labels: number of independent records/number of camera locations where the morph was detected.

camera trapped above 3,600 m (Dhendup 2016). Despite some records of multiple morphs in the same locations (Vernes et al. 2015), several researchers have speculated a possible selection for specific morphs at different altitudes, perhaps as a response to changing vegetation cover with altitude (Sangay et al. 2014, Dhendup 2016). We have amassed a sufficiently large data set of multiple color morphs over a large altitudinal range that allows, for the first time, exploration of empirical evidence for habitat segregation in relation to coat type.

Our data show that there is indeed a statistically significant difference in the elevations where specific color morphs are found (Fig. 1; see Appendix S2 for statistical analyses). Our results confirm previous findings that the golden morph is the most widely distributed form. However, we recorded gray, melanistic, and ocelot morphs over a much wider altitudinal range than previously described. Although the midelevations (1,700-2,500 m) host multiple different morphs in same locations, distribution on the two extremes appears to be more exclusive. Higher elevations $(>3,000 \mathrm{~m})$ show a tendency toward more patterned forms, whereas lower elevations $(<1,700$ m) are dominated by solid-colored morphs (cinnamon, golden, or melanistic). In particular, the distribution ranges of melanistic and tightly rosetted morphs did not overlap. Additionally, different morphs were active at different times of the day. The two patterned morphs (ocelot and tightly rosetted) and the gray morphs were only active at nighttime, compared with the other solid-colored morphs that are active throughout the day (Appendix S3: Fig. S1). Different morphs thus appear to be segregating niches, not just spatially but also temporally.

Previous studies of the adaptive function of feline coat patterns have indicated that they are likely to be for camouflage rather than communication (including aposematism) or physiological reasons (Ortolani and Caro 1996, Caro 2009). Recent work on the distribution of two jaguarundi coloration phenotypes showed that reddish jaguarundis were more common in open habitats, whereas the frequency of gray/dark animals was higher in forested areas (da Silva et al. 2016). A range-wide study on distribution of melanism in leopards found a similar association with closed/moist forests (da Silva et al. 2017). Further, the existence of color polymorphisms in several different feline species (11 of 37 species) suggests that this trait may promote ecological success of species by permitting (via better camouflage) 
use of a wider spectrum of available resources (Forsman et al. 2008).

Dibang Valley hosts a wide range of habitats from wet tropical and subtropical forest in the lower elevations $(<1,700 \mathrm{~m})$ through montane forest in mid altitudes to rhododendron forest, alpine scrub, and high-altitude pasture $(>3,500 \mathrm{~m})$. In addition to elevation, habitat in this mountainous landscape also changes in response to aspect and micro geographical features such as valleys. For a medium-sized predator like the golden cat, camouflage would be a key adaptation to exploit the unique prey assemblage in each of these diverse habitats successfully, whether it is tropical pheasants, rodents, and reptiles in warmer low-elevation forests or Himalayan pika and montane galliformes in the higher elevations. The two patterned morphs were not only more common in higher elevations, which are wetter and mistier (Nijhawan 2018), they were also more nocturnal. This complements earlier findings that irregular patterns in wild felines correlate with more nocturnal hunters and those found in darker/closed environments (Allen et al. 2011). Given that this global cross-species phenomenon also seems to exist within a species at a local scale suggests a more fundamental relationship linking coat type to specific environments.

A greater question now arises: why do golden cats have such a diverse range of coat patterns in Dibang Valley? We know that color variants arise because of genetic drift or random mutation, but persist and become common in a population through natural selection if they provide ecological advantages (Schneider et al. 2015). But what could be the possible benefits of occurring in so many different forms in one area? Camera data from one locality alone are insufficient in solving such an evolutionary puzzle. Nevertheless, we can speculate plausible explanations for the observed phenomenon.

In Dibang Valley, golden cats share space with a range of carnivores from larger predators such as tigers and Asiatic wild dogs to similar-sized clouded leopards, and smaller predators such as marbled and leopard cats. In such a diverse predator community, polymorphism may allow golden cats to avoid competition and intraspecific predation from larger carnivores by exploiting a wider range of available ecological niches. Graipel et al. (2014) found that melanistic oncillas were more active during bright nights than spotted oncillas, suggesting that melanistic individuals could occupy an alternative and wider ecological niche than spotted individuals. Similarly, Kawanishi et al. (2010) suggested that melanistic leopards may be better concealed from socially dominant tigers in the dimly lit rainforests where potential competition for similar-sized prey is high. Perhaps this explains why more color morphs, including the ocelot type, have been recorded in Dibang Valley and Bhutan, where golden cats co-occur with tigers and leopards throughout their range, but not in Sikkim or Nepal, where tigers no longer exist in considerable densities in higher altitudes.

In both Dibang Valley and Bhutan's Jigme Dorji National Park, areas with a similar prey-predator assemblage, the golden cat (all morphs combined) was the most frequently phototrapped predator (Thinley et al. 2015). In Dibang Valley, its photographic detection rates (correlated with density) were 3-4 times higher than those of clouded leopard, marbled cat, and leopard cat - an indication of the species' ecological success in the area (Nijhawan 2018). Our observations therefore seem to suggest that the great diversity of coat types in Asiatic golden cat may allow it to exploit more ecological niches, particularly in an environment full of competitors and predators. This adaptability may contribute to the making the Asiatic golden cat the most ecologically and geographically widespread wild cat in Dibang Valley.

Perhaps Dibang Valley happens to contain a population of the Asiatic golden cat with remarkably high genetic diversity, making it a melting pot of color morphs adapted to its many habitats. But, perhaps, it is a species-level phenomenon also found in other areas with high levels of habitat and co-predator diversity. In order to generate such a hypothesis, future work should investigate patterns of habitat segregation by morphs by pooling records from other Himalayan sites with a similar elevation/habitat range. Finally, combining empirical studies on other polymorphic cats such as bay cat, jaguarundi, and oncilla could lead to a wider hypothesis around ecological benefits of polymorphism in mediumsized cats in competitive environments.

\section{ACKNOWLEDGMENTS}

These observations were made during Sahil Nijhawan's doctoral fieldwork in Dibang Valley, Arunachal Pradesh. We are grateful to Panthera, Wildcats Conservation Alliance (formerly 21st Century Tiger), Chester Zoo Conservation Fund and Ravi Shankaran-Inlaks Shivdasani Foundation for funding the project. Our sincere gratitude to Dr. Joe Smith and Dr. John Goodrich of Panthera for donating camera traps that made the research possible. Comments from Oliver Wearn, Guilherme Ferreira, Paul Barnes, John Pastor, and two anonymous reviewers greatly improved the manuscript. Finally, we are indebted to Idu Mishmi host families and guides in Dibang Valley. This work would not have been possible without their knowledge, hard work, and persistence.

\section{Literature Cited}

Allen, W. L., I. C. Cuthill, N. E. Scott-Samuel, and R. Baddeley. 2011. Why the leopard got its spots: relating pattern development to ecology in felids. Proceedings of the Royal Society B 278:1373-1380.

Bashir, T., T. Bhattacharya, K. Poudyal, and S. Sathyakumar. 2011. Notable observations on the melanistic Asiatic Golden cat (Pardofelis temminckii) of Sikkim, India. NeBIO 2:2-4.

Caro, T. 2009. Contrasting coloration in terrestrial mammals. Philosophical Transactions of the Royal Society B 364:537-548. 
da Silva, L. G. et al. 2017. Mapping black panthers: macroecological modeling of melanism in leopards (Panthera pardus). PLoS ONE 12:e0170378.

da Silva, L. G., T. G. Oliveira, C. B. Kasper, J. J. Cherem, E. A. Moraes, A. Paviolo, and E. Eizirik. 2016. Biogeography of polymorphic phenotypes: mapping and ecological modelling of coat colour variants in an elusive Neotropical cat, the jaguarundi (Puma yagouaroundi). Journal of Zoology 299:295-303.

Dhendup, T. 2016. Status of Asiatic Golden Cat Catopuma temminckii Vigors \& Horsfield, 1827 (Carnivora: Felidae) in Bhutan. Journal of Threatened Taxa 8:8698-8702.

Forsman, A., J. Ahnesjö, S. Caesar, and M. Karlsson. 2008. A model of ecological and evolutionary consequences of color polymorphism. Ecology 89:34-40.

Ghimirey, Y., and P. Pal. 2009. First camera trap image of Asiatic golden cat in Nepal. Cat News 51:19.

Graipel, M. E., L. G. R. Oliveira-Santos, F. V. B. Goulart, M. A. Tortato, P. R. M. Miller, and N. C. Cáceres. 2014. The role of melanism in oncillas on the temporal segregation of nocturnal activity. Brazilian Journal of Biology 74:142-145.

Jigme, K. 2011. Four colour morphs of and the altitudinal record for the Asiatic golden cat in Bhutan. Cat News 55:12-13.

Kawanishi, K., M. E. Sunquist, E. Eizirik, A. J. Lynam, D. Ngoprasert, W. N. Wan Shahruddin, D. M. Rayan, D. S. K. Sharma, and R. Steinmetz. 2010. Near fixation of melanism in leopards of the Malay Peninsula. Journal of Zoology 282:201-206.

McCarthy, J., S. Dahal, T. Dhendup, T. N. E. Gray, S. Mukherjee, H. Rahman, P. Riordan, N. Boontua, and D. Wilcox. 2015. Catopuma temminckii (errata version published in 2016). The IUCN Red List of Threatened Species 2015: e.T4038A97165437.

Nijhawan, S. 2018. Human-animal relations and the role of cultural norms in tiger conservation in the Idu Mishmi of
Arunachal Pradesh, India. Dissertation. University College London, London, UK.

Ortolani, A., and T. M. Caro. 1996. The adaptive significance of color patterns in carnivores: phylogenetic tests of classic hypotheses. Pages 132-188 in J. Gittleman, editor. Carnivore behaviour, ecology and evolution. Volume 2. Cornell University Press, Ithaca, New York, USA.

Sangay, T., R. Rajaratnam, and K. Vernes. 2014. Wildlife camera trapping in the Himalayan Kingdom of Bhutan with recommendations for the future. Pages 87-98 in P. Meek, P. Fleming, G. Ballard, P. Banks, A. Claridge, J. Sanderson, and D. Swann, editors. Camera trapping: wildlife management and research. CSIRO Publishing, Melbourne, Australia.

Schneider, A. et al. 2015. Recurrent evolution of melanism in South American felids. PLoS Genetics 11:e1004892.

Sunquist, M., and F. Sunquist. 2002. Wild cats of the world. University of Chicago Press, Chicago, Illinois, USA.

Thinley, P., S. J. Morreale, P. D. Curtis, J. P. Lassoie, T. Dorji, L. Leki, S. Phuntsho, and N. Dorji. 2015. Diversity, occupancy, and spatio-temporal occurrences of mammalian predators in Bhutan's Jigme Dorji National Park. Bhutan Journal of Natural Resources and Development 2:19-27.

Vernes, K., T. Sangay, R. Rajaratnam, and R. Singye. 2015. Social interaction and co-occurrence of colour morphs of the Asiatic Golden Cat, Bhutan. Cat News 62:18-20.

Additional supporting information may be found in the online version of this article at http://onlinelibrary.wiley.com/doi/ 10.1002/ecy.2768/suppinfo 